\title{
Supplemental File
}

\section{${ }^{40} \mathrm{Ar} /{ }^{39} \mathrm{Ar}$ Geochronology}

Following the analytical methods described in Nagorsen-Rinke et al. (2013) and Dalrymple and Duffield (1988), we used ${ }^{40} \mathrm{Ar} /{ }^{39} \mathrm{Ar}$ incremental heating techniques or a continuous $\mathrm{CO}_{2}$ laser system to date eight samples collected from Oligocene to Miocene volcanic rocks from across the GVGR (Figs. S1, S2, and S3). The resulting age data provide us with timing of volcanism and faulting, and allows us to calculate fault slip rates across the GVGR. ${ }^{40} \mathrm{Ar} /{ }^{39} \mathrm{Ar}$ geochronology samples were prepared and analyzed following the procedures outlined in Supplemental File 1 in Nagorsen-Rinke et al. (2013). Samples reported herein were irradiated at the U.S. Geological Survey, Denver, Colorado, USA, TRIGA (Training, Research, General Atomics) reactor using the Taylor Creek sanidine at $28.444 \mathrm{Ma}$ (Fleck et al., 2019) as a neutron flux monitor. This standard age is equivalent to the Fish Canyon sanidine age of $28.198 \mathrm{Ma}$ calibrated to the astronomical age of the Melilla tephra (Kuiper et al., 2008). Plateau ages are defined by a consecutive series of steps where $\geq 50 \%$ of ${ }^{39} \mathrm{Ar}$ released is within error. Latitude and longitude recorded in the World Geodetic System 1984. Clean separates (either groundmass, groundmass plagioclase, sanidine, or anorthoclase), collected from three hornblende andesite lavas (OMl, Mlf, and Mal), one quartz latite (OMl), one rhyodacite to quartz latite (Mrc), one rhyolite (Mrl), one basalt (OMl), and one volcanic ash (Me) yield either plateau ages or weighted mean ages (Figs. S1, S2, and S3).

The Oligocene-Miocene unit, OMl, yields a plagioclase weighted mean plateau age of 23.06 $\pm 0.03 \mathrm{Ma}$, a plagioclase weighted mean age of $20.98 \pm 0.10 \mathrm{Ma}$, and groundmass weighted mean plateau age of $15.71 \pm 0.03 \mathrm{Ma}$ (Fig. S1). The oldest Miocene units, $\mathrm{Mrc}$ and Mrl, yield sanidine and anorthoclase weighted mean ages of $22.95 \pm 0.04 \mathrm{Ma}$ and $20.14 \pm 0.26 \mathrm{Ma}$, respectively (Fig. S2). Three younger Miocene units, Mlf, Mal, and Me, yield a plagioclase weighted mean plateau age of $18.91 \pm 0.03 \mathrm{Ma}$, a plagioclase weighted mean plateau age of $15.99 \pm 0.05 \mathrm{Ma}$, and an anorthoclase weighted mean age of $11.8 \pm 0.1 \mathrm{Ma}$ (Fig. S3).

\section{References Cited}

Dalrymple, G.B., and Duffield, W.A., 1988, High precision ${ }^{40} \mathrm{Ar} /{ }^{39} \mathrm{Ar}$ dating of Oligocene rhyolites from the Mogollon-Datil volcanic field using a continuous laser system: Geophysical Research Letters, v. 15, p. 366-463.

Fleck, R.J., Calvert, A.T., Coble, M.A., Wooden, J.L., Hodges, K., Hayden, L.A., van Soest, M.C., du Bray, E.A., and John, D.A., 2019, Characterization of the rhyolite of Bodie Hills and ${ }^{40} \mathrm{Ar} /{ }^{39} \mathrm{Ar}$ intercalibration with Ar mineral standards, Chemical Geology, v. 525, p. 282-302

Kuiper, K.F., Deino, A., Hilgen, F.J., Krijgsman, W., Renne, P.R., and Wijbrans, J.R., 2008. Synchronizing rock clocks of Earth history, Science, v. 320, p. 500-504.

Nagorsen-Rinke, S., Lee, J., and Calvert, A., 2013, Pliocene sinistral slip across the Adobe Hills, eastern California-western Nevada: Kinematics of fault slip transfer across the Mina 
deflection: Geosphere, v. 9, no. 1, p. 37-53. 

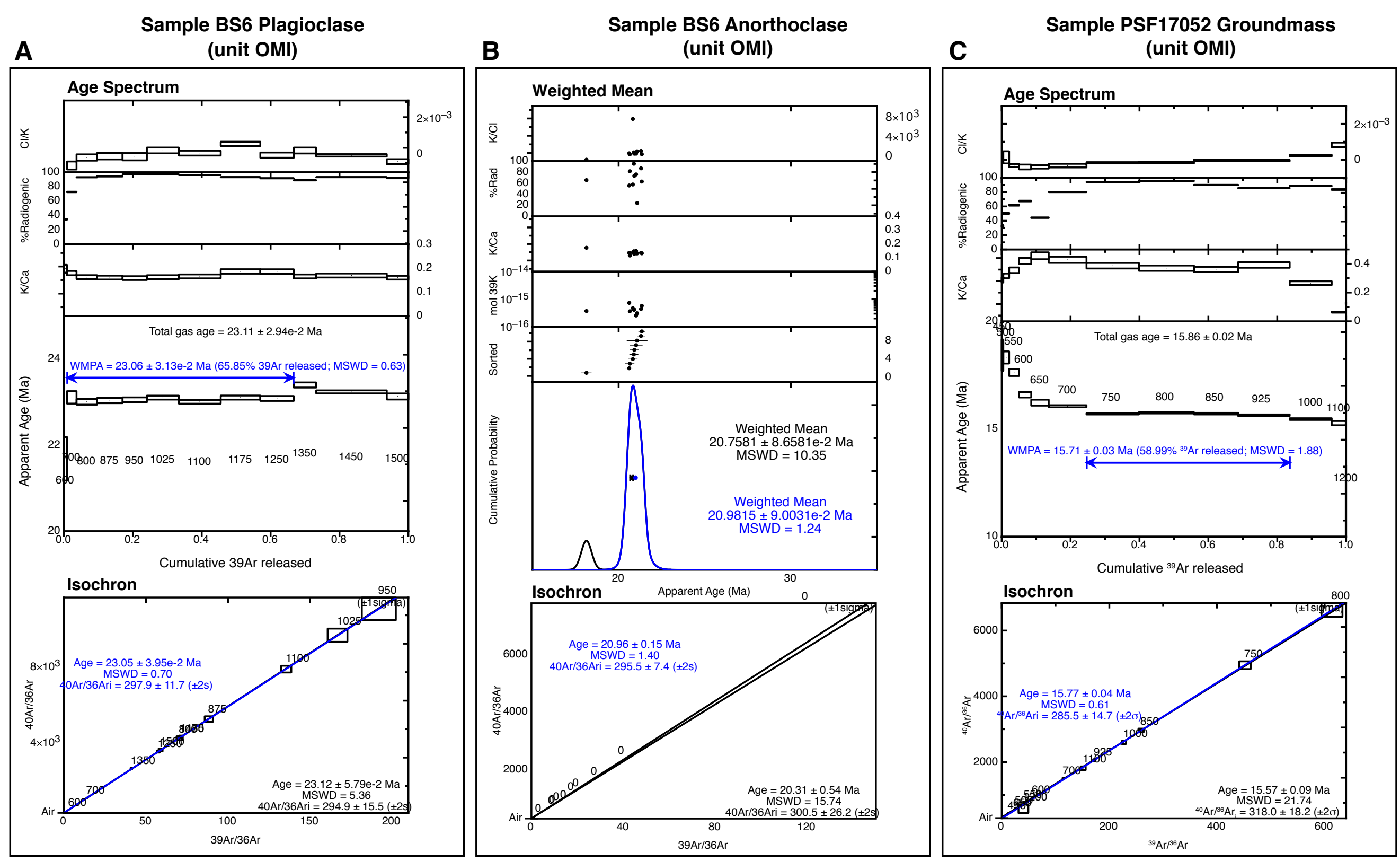

Figure S1. ${ }^{40} \mathrm{Ar} /{ }^{39} \mathrm{Ar}$ age data from the Oligocene-Miocene unit OMl. (A) Age spectrum for plagioclase from a hornblende andesite lava.

(B) age probability distribution plot for anorthoclase from a quartz latite, and (C) age spectrum for groundmass from a basalt lava. Summary of ages is shown in Table 1; analytical data are listed in the Supplemental File. 

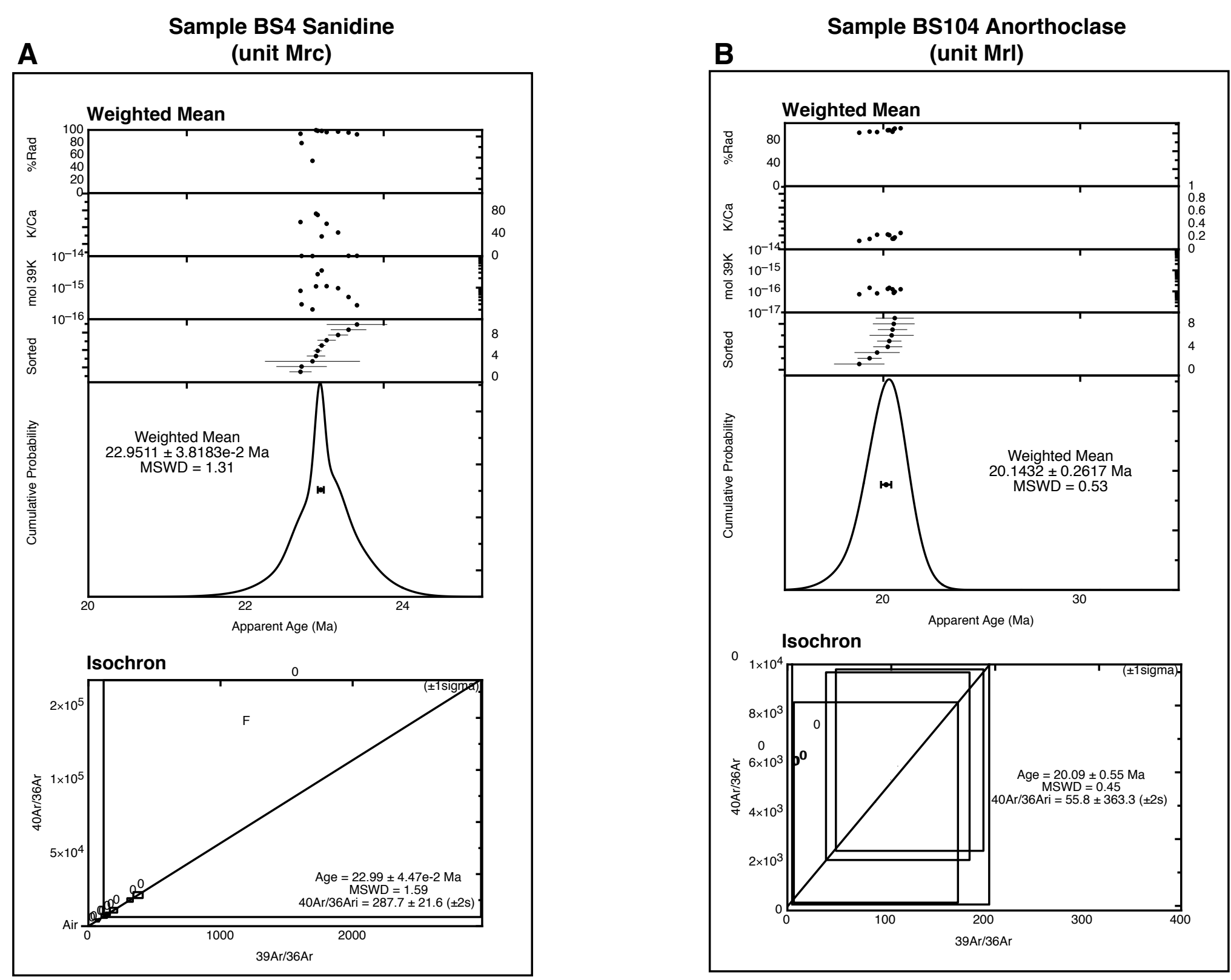

Figure S2. ${ }^{40} \mathrm{Ar} /{ }^{39} \mathrm{Ar}$ age data from the oldest Miocene units, Mrc and Mrl. (A) Age probability distribution plot for sanidine from unit Mrc, and (B) age probability distribution plot for anorthoclase from unit Mrl. Summary of ages is shown in Table 1; analytical data are listed in the Supplemental File. 

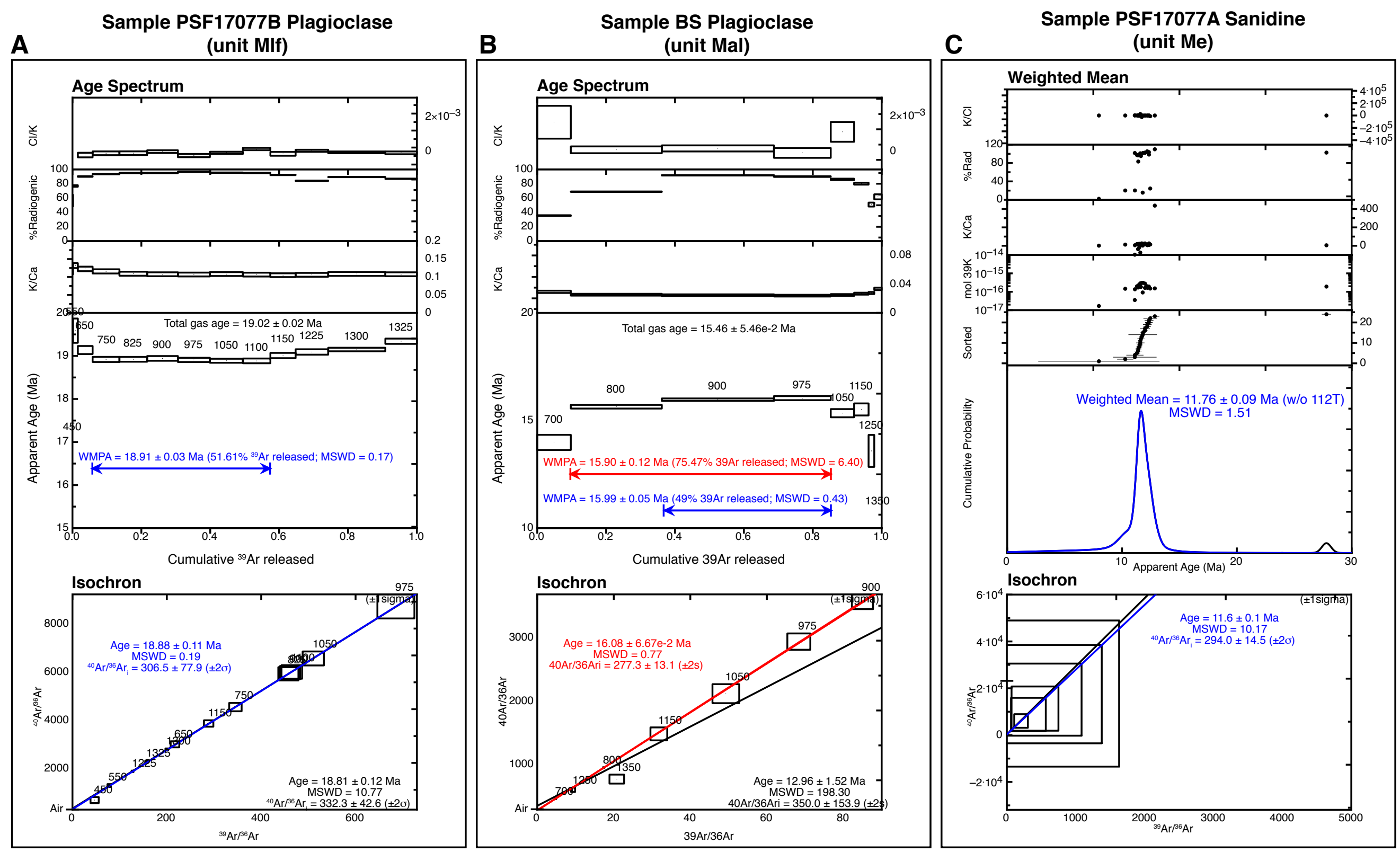

Figure S3. ${ }^{40} \mathrm{Ar} /{ }^{39} \mathrm{Ar}$ age data from the youngest Miocene units, Mlf, Mal, and Me. (A) Age spectrum for plagioclase from unit Mlf, (B) age spectrum for plagiocalse from unit Mal, and (C) age probability distribution plot for anorthoclase from unit Me. Summary of ages is shown in Table 1; analytical data are listed in the Supplemental File. 


\section{Table 40Ar/39Ar tabulated data}

\section{BS10 Anorthoclase (unit Mrl)}

\begin{tabular}{|c|c|c|c|c|c|c|c|c|c|c|}
\hline irain & Age(Ma) & $\% 40 A r *$ & $\mathrm{~K} / \mathrm{Ca}$ & $\mathrm{K} / \mathrm{Cl}$ & moles 40Ar* & $\Sigma 39 \mathrm{Ar}$ & $40 \mathrm{Ar}$ & $39 \mathrm{Ar}$ & $38 \mathrm{Ar}$ & $37 \mathrm{Ar}$ \\
\hline A & $19.30 \pm 0.61$ & 95.17 & 0.16 & -189 & 6. $92 \mathrm{E}-15$ & 0.14 & $0.049132 \pm 0.000056$ & $0.001001 \pm 0.000009$ & $-0.000008 \pm 0.000010$ & $0.003178 \pm 0.0000$ \\
\hline & $19.68 \pm 1.15$ & 94.43 & 0.23 & -519 & $3.84 \mathrm{E}-15$ & 0.21 & $0.027480 \pm 0.000055$ & $0.000544 \pm 0.000009$ & $0.000004 \pm 0.000010$ & $0.001233 \pm 0.000$ \\
\hline & $20.30 \pm 0.62$ & 97.77 & 0.22 & -871 & $7.24 \mathrm{E}-15$ & 0.34 & $50008 \pm 0.000082$ & $0.000994 \pm 0.000010$ & $0.000009 \pm c$ & \\
\hline & $20.47 \pm 0.75$ & 94.96 & 0.17 & -584 & $6.24 \mathrm{E}-15$ & 0.46 & 0071 & $0.000850 \pm 0$. & $0.000006 \pm c$ & 0.00 \\
\hline & $20.22 \pm 0.74$ & 97.58 & 0.24 & -3427 & & & & & & \\
\hline & $20.53 \pm 1.06$ & 98.86 & 0.17 & -551 & $4.21 \mathrm{E}-15$ & 0.66 & $0.028803 \pm 0.000064$ & $0.000573 \pm 0.000008$ & $0.000003 \pm 0.000007$ & $0.001782 \pm 0.0$ \\
\hline & $20.58 \pm 0.97$ & 100.56 & 0.19 & -194 & 4. $79 \mathrm{E}-15$ & 0.74 & $0.032160 \pm 0.000064$ & $0.000649 \pm 0.000009$ & $-0.000006 \pm 0.000010$ & $0.001805 \pm 0.00$ \\
\hline & $20.88 \pm 0.77$ & 101.06 & 0.26 & -258 & 6. 39E-15 & 0.8 & $0.042700 \pm 0.000074$ & $0.000853 \pm 0.000011$ & $-0.000003 \pm 0.000010$ & \\
\hline & & & & & & & & \pm 0.00 & $000 \pm 0.000009$ & \\
\hline
\end{tabular}

Packet IRR348-LS, Experiment \# 16Z0067, xx g San, all errors \pm 1 sigma

$\mathrm{J}=0.000229669620 \pm 0.000000247142$

40Ar* is radiogenic argon, isotopes in volts $(1.48 \mathrm{e}-13$ moles/volt), corrected for blank, background, discrimination, and decay

Calculated bulk K/Ca $=0.189 \pm 5.509 \mathrm{e}-2$, Calculated K2O = INF\%wt. Calculated $\mathrm{CaO}=\mathrm{INF} \%$ wt. , Calculated Cl $=-\mathrm{INFppm}$

Total Gas Age $=20.15 \pm 0.26 \mathrm{Ma}$

\section{Weighted Mean Plateau Age $=20.14 \pm 0.26 \mathrm{Ma}( \pm 1$ sigma, including $\pm \mathrm{J}), \mathbf{1 0 0 . 0 0 \%} 39 \mathrm{Ar}$ released}

Weighted Mean Plateau Age $=20.14 \pm 0.26 \mathrm{Ma}$ (A priori, including $\pm \mathrm{J}), 100.00 \% 39 \mathrm{Ar}$ released

Weighted Mean Plateau Age $=20.14 \pm 0.44 \mathrm{Ma}(95 \%$ confidence, including $\pm \mathrm{J})$

MSWD $=0.53$ (Good fit, MSWD < 2.11)

Grains 10 of 10

Isochron Age $=20.1 \pm 0.6 \mathrm{Ma}( \pm 1$ sigma, including $\pm \mathrm{J})$

Isochron Age $=20.1 \pm 0.6 \mathrm{Ma}$ (A Priori Errors, including $\pm \mathrm{J}$ )

Isochron Age $=20.1 \pm 1.3 \mathrm{Ma}(95 \%$ confidence, including $\pm \mathrm{J})$

MSWD $=0.45$ (Good fit, MSWD < 2.19)

$40 \mathrm{Ar} / 36 \mathrm{Ar}$ intercept $=55.8 \pm 153.6( \pm 1$ sigma $)$

40Ar/36Ar intercept $=55.8 \pm 153.6$ (A Priori)

$40 \mathrm{Ar} / 36 \mathrm{Ar}$ intercept $=55.8 \pm 363.3$ (95\% confidence)

Grains 10 of 10

\section{BS4 Sanidine (unit Mrc)}

\begin{tabular}{|c|c|c|c|c|c|c|c|c|c|c|}
\hline Grain & Age(Ma) & $40 A r *$ & $\mathrm{~K} / \mathrm{Ca}$ & $\mathrm{K} / \mathrm{Cl}$ & moles 40Ar* & $\sum 39 \mathrm{Ar}$ & $40 \mathrm{Ar}$ & $39 \mathrm{Ar}$ & $38 \mathrm{Ar}$ & $37 \mathrm{Ar}$ \\
\hline A & $22.85 \pm 0.60$ & 51.07 & 0.17 & 576 & 1. $15 \mathrm{E}-14$ & 0.02 & $20.151435 \pm 0.000163$ & $0.001385 \pm 0.000020$ & $0.000075 \pm 0.000009$ & $0.004268 \pm 0.0000$ \\
\hline B & $23.41 \pm 0.38$ & 92.99 & 0.53 & -1547 & $1.59 \mathrm{E}-14$ & 0.04 & $40.115523 \pm 0.000129$ & $0.001874 \pm 0.000016$ & $0.000025 \pm 0.000010$ & $0.001856 \pm 0.0000$ \\
\hline & $23.17 \pm 0.13$ & 97.34 & 41.92 & 2027 & $5.45 \mathrm{E}-14$ & 0.13 & $30.378482 \pm 0$ & $0.006488 \pm 0$ & $0.000105 \pm 0$. & \\
\hline & $23.03 \pm 0.12$ & 96.58 & 57.51 & 2126 & $6.30 \mathrm{E}-14$ & 0.22 & 20.4 & 026 & 009 & גדנה \\
\hline & $23.30 \pm 0.23$ & & 0.52 & -40032 & E-14 & 0.27 & & 0.00 & 012 & \\
\hline & $22.89 \pm 0.12$ & 9.66 & 75.36 & 3047 & $E-14$ & & & 030 & & \\
\hline & $22.71 \pm 0.32$ & 79.23 & 0.33 & 2186 & $1.65 \mathrm{E}-14$ & 0.39 & $90.140864 \pm 0.000117$ & $0.002008 \pm 0.000012$ & $0.000049 \pm$ & $0.003190 \pm 0$ \\
\hline & $22.91 \pm 0.05$ & 98.62 & 73.15 & 13168 & 1. $50 \mathrm{E}-13$ & 0.62 & $33 \pm 0.000472$ & $0.018099 \pm 0.000032$ & $0.000253 \pm 0.000015$ & $0.000130 \pm 0.000 c$ \\
\hline & $22.69 \pm 0.14$ & & 60.53 & 1227 & 4. $42 \mathrm{E}-14$ & & $90.318106 \pm 0.000179$ & & & \\
\hline & $22.96 \pm 0.05$ & 98.36 & 35.01 & 3014 & 1. $97 \mathrm{E}-13$ & & $11.353322 \pm 0.000556$ & $0.023656 \pm 0.000040$ & $0.000359 \pm 0.000014$ & $0.000355 \pm 0.00$ \\
\hline
\end{tabular}

$36 \mathrm{Ar}$

$0.000252 \pm 0.00000$ $0.000028 \pm 0.000005$ $0.000034 \pm 0.000005$ $0.000051 \pm 0.000005$ $0.000029 \pm 0.000005$ $0.000005 \pm 0.000004$ $0.000005 \pm 0.000004$ $0.000100 \pm 0.000005$ $0.000048 \pm 0.000005$ $0.000075 \pm 0.000005$

, xx g San, all errors \pm 1 sigma

$\mathrm{J}=0.000227632520 \pm 0.000000211219$

40Ar* is radiogenic argon, isotopes in volts (1.48e-13 moles/volt), corrected for blank, background, discrimination, and decay

Calculated bulk K/Ca $=3.008 \pm 1.138$, Calculated K2O $=$ INF\%wt., Calculated $\mathrm{CaO}=\mathrm{INF} \%$ wt. , Calculated $\mathrm{Cl}=\mathrm{INFppm}$ 
Weighted Mean Age $=22.95 \pm$ 4.24e-2 Ma ( \pm 1 sigma, including $\pm J), 100.00 \% 39 \mathrm{Ar}$ released

Weighted Mean Age $=22.95 \pm 3.65 \mathrm{e}-2 \mathrm{Ma}$ (A priori, including $\pm \mathrm{J}), 100.00 \% 39 \mathrm{Ar}$ released

Weighted Mean Age $=22.95 \pm 9.78 \mathrm{e}-2 \mathrm{Ma}(95 \%$ confidence, including $\pm \mathrm{J}$ )

MSWD $=1.35($ Good fit, MSWD $<2.11)$

10 of 10 grains

Isochron Age $=22.99 \pm 0.04 \mathrm{Ma}( \pm 1$ sigma, including $\pm \mathrm{J})$

Isochron Age $=23.0 \pm 0.05 \mathrm{Ma}$ (A Priori Errors, including $\pm \mathrm{J}$ )

Isochron Age $=23.0 \pm 0.1 \mathrm{Ma}(95 \%$ confidence, including $\pm \mathrm{J})$

MSWD $=1.59$ (Good fit, MSWD $<2.19$ )

$40 \mathrm{Ar} / 36 \mathrm{Ar}$ intercept $=287.7 \pm 9.1( \pm 1$ sigma $)$

40Ar/36Ar intercept $=287.7 \pm 7.2$ (A Priori)

$40 \mathrm{Ar} / 36 \mathrm{Ar}$ intercept $=287.7 \pm 21.6(95 \%$ confidence $)$

10 of 10 grains

\section{BS6 Anorthoclase (quartz latite flow in unit $\mathrm{OMI}$ )}

\begin{tabular}{|c|c|c|c|c|c|c|c|c|c|c|c|}
\hline Grain & Age(Ma) & $\% 40 A r *$ & $\mathrm{~K} / \mathrm{Ca}$ & $\mathrm{K} / \mathrm{Cl}$ & moles 40Ar* & $\Sigma 39 \mathrm{Ar}$ & $40 \mathrm{Ar}$ & $39 \mathrm{Ar}$ & $38 \mathrm{Ar}$ & $37 \mathrm{Ar}$ & $36 \mathrm{Ar}$ \\
\hline & $21.01 \pm 0.38$ & 75.91 & 0.15 & 488 & $1.32 \mathrm{E}-14$ & 0.06 & $60.117304 \pm 0.000083$ & $0.001741 \pm 0.000012$ & $0.000056 \pm 0.000012$ & $0.006103 \pm 0.000076$ & $0.000097 \pm 0.000005$ \\
\hline B & $21.35 \pm 0.20$ & 63.01 & 0.13 & 375 & 3. $01 \mathrm{E}-14$ & 0.19 & $90.322966 \pm 0.000188$ & $0.003918 \pm 0.000016$ & $0.000172 \pm 0.000012$ & $0.015490 \pm 0.000099$ & $0.000409 \pm 0.000006$ \\
\hline C & $20.65 \pm 0.26$ & 81.69 & 0.12 & 338 & $1.84 \mathrm{E}-14$ & 0.27 & $0.152129 \pm 0.000141$ & $0.002474 \pm 0.000015$ & $0.000082 \pm 0.000009$ & $0.011126 \pm 0.000086$ & $0.000097 \pm 0.000005$ \\
\hline D & $20.91 \pm 0.25$ & 73.43 & 0.13 & 484 & $14 \mathrm{E}-14$ & $0.37-3$ & $70.196438 \pm 0.000165$ & $0.002835 \pm 0.000015$ & $0.000096 \pm 0.0$ & 00072 & 0005 \\
\hline $\mathrm{E}$ & $21.30 \pm 0.22$ & 86.96 & 0.14 & 1028 & 2. $29 \mathrm{E}-14$ & 0.47 & $70.177845 \pm 0.000150$ & $0.002983 \pm 0.000016$ & $0.000067 \pm 0$ & 0111 & $0.000082 \pm 0$ \\
\hline $\mathrm{F}$ & $20.62 \pm 0.23$ & 55.89 & 0.14 & 557 & 3. $71 \mathrm{E}-14$ & 0.6 & $40.448552 \pm 0.000249$ & $0.004997 \pm 0.000021$ & $0.000230 \pm 0$ & 0.01 & $0.000675 \pm 0$. \\
\hline G & & 95. & 0.15 & 742 & 2E-14 & & & & & & 006 \\
\hline $\mathrm{H}$ & $20.82 \pm 0.27$ & 57.69 & 0.14 & 7409 & $2.41 \mathrm{E}-14$ & 0.8 & $40.281910 \pm 0.000184$ & $0.003209 \pm 0.000016$ & $0.000120 \pm 0.0$ & $0.012254 \pm 0.000103$ & $0.000407 \pm 0$ \\
\hline & $18.13 \pm 0.30$ & 65.62 & 0.17 & -715 & $1.65 \mathrm{E}-14$ & 0.93 & $30.169690 \pm 0.000111$ & $0.002524 \pm 0.000015$ & $0.000055 \pm 0.000012$ & $0.007665 \pm 0.000032$ & $0.000200 \pm 0.000006$ \\
\hline & $21.07 \pm 0.60$ & 24.15 & 0.13 & 954 & $1.60 \mathrm{E}-14$ & & $10.446438 \pm 0.000294$ & $0.002102 \pm 0.000017$ & $0.000251 \pm 0.000011$ & $0.008364 \pm 0.000067$ & $0.001148 \pm 0.000010$ \\
\hline
\end{tabular}

Packet IRR348-MC, Experiment \#16Z0070, xx g San, all errors \pm 1 sigma

$\mathrm{J}=0.000228489670 \pm 0.00000023252$

40Ar* is radiogenic argon, isotopes in volts (1.48e-13 moles/volt), corrected for blank, background, discrimination, and decay

Calculated bulk K/Ca $=0.137 \pm 4.530 \mathrm{e}-2$, Calculated $\mathrm{K} 2 \mathrm{O}=\mathrm{INF} \%$ wt. , Calculated $\mathrm{CaO}=\mathrm{INF} \%$ wt. , Calculated $\mathrm{Cl}=\mathrm{INFppm}$

Total Gas Age $=20.70 \pm 0.09 \mathrm{Ma}$

Weighted Mean Age $=20.98 \pm 0.10 \mathrm{Ma}( \pm 1$ sigma, including $\pm \mathrm{J}), 91.47 \%$ 39Ar released

Weighted Mean Age $=20.98 \pm 8.97 \mathrm{e}-2 \mathrm{Ma}$ (A priori, including $\pm \mathrm{J}), 91.47 \% 39 \mathrm{Ar}$ released

Weighted Mean Age $=20.98 \pm 0.23 \mathrm{Ma}(95 \%$ confidence, including \pm J)

MSWD = 1.25 (Good fit, MSWD < 2.19)

9 of 10 grains

Isochron Age $=20.96 \pm 0.15 \mathrm{Ma}( \pm 1$ sigma, including $\pm \mathrm{J})$

Isochron Age $=21.0 \pm 0.1 \mathrm{Ma}$ (A Priori Errors, including $\pm \mathrm{J}$ )

Isochron Age $=21.0 \pm 0.3 \mathrm{Ma}(95 \%$ confidence, including $\pm \mathrm{J})$

MSWD $=1.40$ (Good fit, MSWD < 2.29)

$40 \mathrm{Ar} / 36 \mathrm{Ar}$ intercept $=295.5 \pm 3.1( \pm 1$ sigma $)$

$40 \mathrm{Ar} / 36 \mathrm{Ar}$ intercept $=295.5 \pm 2.6$ (A Priori)

$40 \mathrm{Ar} / 36 \mathrm{Ar}$ intercept $=295.5 \pm 7.4(95 \%$ confidence $)$ 


\section{BS8 Plagioclase (unit Mal)}

\begin{tabular}{|c|c|c|c|c|c|c|c|c|}
\hline $\mathrm{mp}\left({ }^{\mathrm{c}} \mathrm{Age}(\mathrm{Ma})\right.$ & $\% 40 A r^{*}$ & $\mathrm{~K} / \mathrm{Ca}$ & $\mathrm{K} / \mathrm{Cl}$ & moles 40Ar* & $\Sigma 39 \mathrm{Ar}$ & $40 \mathrm{Ar}$ & $39 \mathrm{Ar}$ & $38 \mathrm{Ar}$ \\
\hline $70013.98 \pm 0.35$ & 35.6 & 0.03 & 614 & . $\quad 3.48 \mathrm{E}-14$ & 0.1 & $10.660408 \pm 0.000900$ & $0.007052 \pm 0.000147$ & $0.000413 \pm 0.000027$ \\
\hline $80015.64 \pm 0.09$ & 68.91 & 0.03 & 11024 & 1. $06 \mathrm{E}-13$ & 0.36 & $61.035334 \pm 0.000289$ & $0.019161 \pm 0.000058$ & $0.000472 \pm 0.0000$ \\
\hline $90015.97 \pm 0.06$ & 91.73 & 0.02 & 5902 & 1. $33 \mathrm{E}-13$ & 0.69 & $90.978562 \pm 0.000321$ & $0.023623 \pm 0.000042$ & $0.000390 \pm 0.000$ \\
\hline $97516.04 \pm 0.09$ & 89.96 & 0.02 & -12386 & $6.76 \mathrm{E}-14$ & 0.85 & $50.507422 \pm 0.000247$ & $0.011963 \pm 0.000036$ & $0.000191 \pm 0.0$ \\
\hline $105015.34 \pm 0.19$ & 86.07 & 0.02 & 916 & 2. $66 \mathrm{E}-14$ & 0.92 & $20.208939 \pm 0.000174$ & $0.004926 \pm 0.000023$ & \\
\hline $115015.51 \pm 0.28$ & 80.12 & 0.03 & 308 & $1.66 \mathrm{E}-14$ & 0.96 & $60.140247 \pm 0.000123$ & $0.003040 \pm 0.000018$ & $0.000101 \pm 0.00$ \\
\hline $125013.59 \pm 0.74$ & 50.97 & 0.03 & 37 & $5.73 \mathrm{E}-15$ & 0.98 & $80.075943 \pm 0.000117$ & $0.001196 \pm 0.000012$ & $0.000177 \pm 0.00$ \\
\hline $13509.51 \pm 0.55$ & 61.74 & 0.03 & 204 & 5. $22 \mathrm{E}-15$ & & $10.057140 \pm 0.000104$ & $0.001555 \pm 0.000012$ & $0.000067 \pm 0.00$ \\
\hline
\end{tabular}

$.124489 \pm 0.00050$ $0.390821 \pm 0.00068$ $0.496134 \pm 0.000598$ $0.257328 \pm 0.000559$ $0.102129 \pm 0.000434$ $0.058711+0.000262$ $0.058328 \pm 0.000$ $0.022328 \pm 0.000198$ $\begin{array}{lllll}1 & 0.057140 \pm 0.000104 & 0.001555 \pm 0.000012 & 0.000067 \pm 0.000011 & 0.024120 \pm 0.000214\end{array}$ Packet IRR348-LV, Experiment \#16Z0109, $0.0858 \mathrm{~g}$ Sanidine, all errors \pm 1 sigma

$=0.000230468333 \pm 0.000000253305$

40Ar* is radiogenic argon, isotopes in volts (1.48e-13 moles/volt), corrected for blank, background, discrimination, and decay

Calculated bulk K/Ca $=2.540 \mathrm{e}-2 \pm 1.199 \mathrm{e}-2$, Calculated K2O $=0.21 \%$ wt., Calculated $\mathrm{CaO}=9.92 \%$ wt., Calculated $\mathrm{Cl}=0.2 \mathrm{ppm}$

Total Gas Age $=15.46 \pm 5.46 \mathrm{e}-2 \mathrm{Ma}$

Weighted Mean Plateau Age $=15.90 \pm 0.12 \mathrm{Ma}( \pm 1$ sigma, including $\pm \mathrm{J}), 75.47 \% 39 \mathrm{Ar}$ released

Weighted Mean Plateau Age $=15.90 \pm 4.59 \mathrm{e}-2 \mathrm{Ma}$ (A priori, including \pm J), $75.47 \% 39 \mathrm{Ar}$ released

Weighted Mean Plateau Age $=15.90 \pm 0.28 \mathrm{Ma}(95 \%$ confidence, including $\pm \mathrm{J})$

MSWD $=6.40$ (Poor fit, MSWD > 3.69)

Steps 3 of $8\left(800,900,975^{\circ} \mathrm{C}\right)$

Isochron Age $=16.08 \pm 0.07 \mathrm{Ma}( \pm 1$ sigma, including $\pm \mathrm{J})$

Isochron Age $=16.08 \pm 0.07 \mathrm{Ma}$ (A Priori Errors, including $\pm \mathrm{J}$ )

Isochron Age $=16.1 \pm 0.2 \mathrm{Ma}(95 \%$ confidence, including $\pm \mathrm{J})$

MSWD $=0.77$ (Good fit, MSWD < 5.02)

40Ar/36Ar intercept $=277.3 \pm 5.1( \pm 1$ sigma $)$

40Ar/36Ar intercept $=277.3 \pm 5.1$ (A Priori)

40Ar/36Ar intercept $=277.3 \pm 13.1$ (95\% confidence)

Steps 3 of $8\left(800,900,975^{\circ} \mathrm{C}\right)$

\section{Weighted Mean Plateau Age $=15.99 \pm 0.05 \mathrm{Ma}( \pm 1$ sigma, including $\pm J), 49.0 \% 39 \mathrm{Ar}$ released}

Weighted Mean Plateau Age $=15.99 \pm 0.05 \mathrm{Ma}$ (A priori, including $\pm \mathrm{J}), 49.0 \% 39 \mathrm{Ar}$ released

Weighted Mean Plateau Age $=15.87 \pm 0.62 \mathrm{Ma}(95 \%$ confidence, including $\pm \mathrm{J})$

MSWD $=0.43($ Good fit, MSWD $<2.77)$

Steps 2 of $8\left(900,975^{\circ} \mathrm{C}\right)$

\section{BS5 Plagioclase (hornblende andesite lava in unit OMI)}

$60021.68 \pm 0.51$

$70023.11 \pm 0.14$

A $34 . \mathrm{Ca} \mathrm{K} / \mathrm{Cl}$

$\begin{array}{rrr}34.53 & 0.2 & -122 \\ 72.64 & 0.18 & -1609 \\ 93 & 0.16 & -5672\end{array}$

moles 40Ar*

¿39Ar 40Ar

$39 \mathrm{Ar}$

$38 \mathrm{Ar}$

$\begin{array}{lll}93 & 0.16 & -5672\end{array}$

2. $65 \mathrm{E}-14$

$\begin{array}{lll}0.01 & 0.519015 \pm 0.000226 & 0.003414 \pm 0.000052 \\ 0.04 & 0.829745 \pm 0.000248 & 0.010772 \pm 0.000043\end{array}$

$1.90 \mathrm{E}-13$

$95023.05 \pm 0.06$

$97.32 \quad 0.16 \quad-7481$ $\begin{array}{llll}0.1 & 1.383132 \pm 0.000377 & 0.023097 \pm 0.000051 & 0.000349 \pm 0.000017\end{array}$

$\begin{array}{llll}0.17 & 1.704621 \pm 0.000472 & 0.028844 \pm 0.000056 & 0.000429 \pm 0.000022\end{array}$

$\begin{array}{llll}0.24 & 1.572171 \pm 0.000406 & 0.027420 \pm 0.000053 & 0.000373 \pm 0.000024\end{array}$
37Ar

$0.009078 \pm 0.000584$

$0.031541 \pm 0.000316$

$0.074696 \pm 0.000527$

$0.094391 \pm 0.000898$

$0.090944 \pm 0.00032$
$36 \mathrm{Ar}$

$0.001474 \pm 0.00001$

$0.001199 \pm 0.000010$

$0.000413 \pm 0.000008$

$0.000245 \pm 0.000007$

$0.000127 \pm 0.000007$

$0.000111 \pm 0.000006$

$0.000132 \pm 0.000007$

$0.000081 \pm 0.000007$ 
$102523.10 \pm 0.05$ $110023.01 \pm 0.04$ $117523.10 \pm 0.05$ $125023.08 \pm 0.06$ $135023.40 \pm 0.06$ $145023.24 \pm 0.03$ $150023.13 \pm 0.07$

$\begin{array}{lll}96.94 & 0.16 & 4614\end{array}$ $\begin{array}{rrr}96.24 & 0.16 & 14690\end{array}$ $\begin{array}{lll}93.12 & 0.19 & 1715\end{array}$ $\begin{array}{llll}91.6 & 0.19 & -22375\end{array}$ $\begin{array}{lll}88.76 & 0.16 & 4706\end{array}$ $93.11 \quad 0.17 \quad-17303$ $91.8 \quad 0.16 \quad-2453$ $\begin{array}{llll}0.33 & 2.083755 \pm 0.000700 & 0.036116 \pm 0.000057 & 0.000551 \pm 0.000025\end{array}$ \begin{tabular}{llll} 
& \\
\hline
\end{tabular} $\begin{array}{llll}0.57 & 2.683664 \pm 0.000964 & 0.044668 \pm 0.000074 & 0.000818 \pm 0.000023\end{array}$

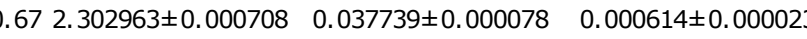
$\begin{array}{llll}0.73 & 1.615186 \pm 0.000398 & 0.025311 \pm 0.000050 & 0.000472 \pm 0.000018\end{array}$

$\begin{array}{llll}1.94 & 4.808718 \pm 0.001079 & 0.079592 \pm 0.000102 & 0.001241 \pm 0.000023\end{array}$

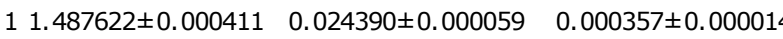

$0.116882 \pm 0.000346$ $0.149806 \pm 0.000859$ $0.126036 \pm$ $0.106726 \pm 0$

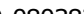
$0.247044 \pm 0.000517$ $0.079999 \pm 0.000320$
$0.000249 \pm 0.000008$ $0.000390 \pm 0.000008$ $0.000660 \pm 0.000010$ $0.000685 \pm 0.000009$ $0.000637 \pm 0.000008$ $0.001190 \pm 0.00001$ $0.000435 \pm 0.000008$

$\mathrm{J}=0.000229914529 \pm 0.000000245114$

40Ar* is radiogenic argon, isotopes in volts (1.48e-13 moles/volt), corrected for blank, background, discrimination, and decay

Calculated bulk K/Ca $=0.168 \pm 5.660 \mathrm{e}-2$, Calculated K2O $=0.91 \%$ wt., Calculated $\mathrm{CaO}=6.64 \%$ wt., Calculated $\mathrm{Cl}=-5.1 \mathrm{e}-2 \mathrm{ppm}$

Total Gas Age $=23.11 \pm 0.02 \mathrm{Ma}$

\section{Weighted Mean Plateau Age $=23.06 \pm 3.13 e-2 \mathrm{Ma}( \pm 1$ sigma, including $\pm \mathrm{J}), 65.85 \% 39 \mathrm{Ar}$ released}

Weighted Mean Plateau Age $=23.06 \pm 3.13 \mathrm{e}-2 \mathrm{Ma}$ (A priori, including $\pm \mathrm{J}), 65.85 \% 39 \mathrm{Ar}$ released

Weighted Mean Plateau Age $=23.06 \pm 5.53 \mathrm{e}-2 \mathrm{Ma}(95 \%$ confidence, including $\pm \mathrm{J})$

MSWD $=0.63$ (Good fit, MSWD < 2.29)

Steps 8 of $12\left(700,800,875,950,1025,1100,1175,1250^{\circ} \mathrm{C}\right)$

Isochron Age $=23.05 \pm 0.04 \mathrm{Ma}( \pm 1$ sigma, including $\pm \mathrm{J})$

Isochron Age $=23.05 \pm 0.0 \mathrm{Ma}$ (A Priori Errors, including $\pm \mathrm{J})$

Isochron Age $=23.05 \pm 0.1 \mathrm{Ma}(95 \%$ confidence, including $\pm \mathrm{J})$

MSWD $=0.70$ (Good fit, MSWD $<2.40$ )

40Ar/36Ar intercept $=297.9 \pm 5.2( \pm 1$ sigma $)$

$40 \mathrm{Ar} / 36 \mathrm{Ar}$ intercept $=297.9 \pm 5.2$ (A Priori)

40Ar/36Ar intercept $=297.9 \pm 11.7$ (95\% confidence)

Steps 8 of $12\left(700,800,875,950,1025,1100,1175,1250^{\circ} \mathrm{C}\right)$

\section{PSF17077A Sanidine (unit Me)}

\begin{tabular}{|c|c|c|c|c|c|c|c|c|c|c|c|}
\hline Grain & $\mathrm{Age}(\mathrm{Ma})$ & $\% 40 A r^{*}$ & $\mathrm{~K} / \mathrm{Ca}$ & $\mathrm{K} / \mathrm{Cl}$ & moles 40Ar* & $\sum 39 \mathrm{Ar}$ & $40 \mathrm{Ar}$ & $39 \mathrm{Ar}$ & $38 \mathrm{Ar}$ & $37 \mathrm{Ar}$ & $36 \mathrm{Ar}$ \\
\hline A & $11.12 \pm 0.75$ & 20.3 & 5.8 & 2201 & $3.63 \mathrm{E}-15$ & 0.03 & $0.251974 \pm 0.000201$ & $0.001920 \pm 0.000023$ & $0.000155 \pm 0.000020$ & $0.000173 \pm 0.000144$ & $0.000673 \pm 0.000011$ \\
\hline B & $10.28 \pm 0.70$ & 20.2 & 12.8 & -174 & 3. 59E-15 & 0.06 & $0.249562 \pm 0.000213$ & $0.002052 \pm 0.000019$ & $0.000101 \pm 0.000014$ & $0.000084 \pm 0.000075$ & $0.000667 \pm 0.000011$ \\
\hline C & $11.80 \pm 1.24$ & 15.6 & 2.0 & -405 & $2.58 \mathrm{E}-15$ & 0.08 & $0.233282 \pm 0.000190$ & $0.001288 \pm 0.000018$ & $0.000127 \pm 0.000019$ & $0.000336 \pm 0.000135$ & $0.000660 \pm 0.000013$ \\
\hline D & $7.98 \pm 5.28$ & 2.1 & 1.3 & -239 & 3. $22 \mathrm{E}-16$ & 0.09 & $0.213452 \pm 0.000215$ & $0.000238 \pm 0.000011$ & $0.000130 \pm 0.000018$ & $0.000098 \pm 0.000237$ & $0.000700 \pm 0.000010$ \\
\hline $\mathrm{E}$ & $12.45 \pm 0.59$ & 24.5 & 14.0 & -11683 & 4. $46 \mathrm{E}-15$ & 0.12 & $0.254123 \pm 0.000260$ & $0.002092 \pm 0.000021$ & $0.000147 \pm 0.000017$ & $0.000078 \pm 0.000117$ & $0.000643 \pm 0.000010$ \\
\hline $\mathrm{F}$ & $12.00 \pm 0.42$ & 102.1 & 25.4 & -522 & $4.68 \mathrm{E}-15$ & 0.16 & $0.064375 \pm 0.000123$ & $0.002292 \pm 0.000025$ & $0.000010 \pm 0.000018$ & $0.000047 \pm 0.000200$ & $-0.000005 \pm 0.000007$ \\
\hline G & $11.59 \pm 0.37$ & 101.0 & 4.5 & -631 & $5.21 \mathrm{E}-15$ & 0.2 & $0.072478 \pm 0.000129$ & $0.002643 \pm 0.000023$ & $0.000016 \pm 0.000018$ & $0.000307 \pm 0.000214$ & $-0.000002 \pm 0.000008$ \\
\hline $\mathrm{H}$ & $11.11 \pm 1.90$ & 101.7 & -96.8 & 677 & $9.44 \mathrm{E}-16$ & 0.21 & $0.013041 \pm 0.000067$ & $0.000499 \pm 0.000012$ & $0.000010 \pm 0.000016$ & $-0.000003 \pm 0.000258$ & $-0.000001 \pm 0.000008$ \\
\hline I & $11.83 \pm 0.23$ & 101.9 & 22.8 & -1412 & 8. $70 \mathrm{E}-15$ & 0.28 & $0.119899 \pm 0.000164$ & $0.004321 \pm 0.000031$ & $0.000042 \pm 0.000017$ & $0.000099 \pm 0.000215$ & $-0.000008 \pm 0.000007$ \\
\hline J & $12.35 \pm 0.39$ & 98.3 & 25.6 & -581 & $5.02 \mathrm{E}-15$ & 0.32 & $0.071704 \pm 0.000212$ & $0.002388 \pm 0.000020$ & $0.000014 \pm 0.000017$ & $0.000049 \pm 0.000129$ & $0.000004 \pm 0.000007$ \\
\hline $\mathrm{K}$ & $11.55 \pm 0.25$ & 98.0 & 21.7 & -4651 & $7.05 E-15$ & 0.37 & $0.101081 \pm 0.000176$ & $0.003590 \pm 0.000027$ & $0.000045 \pm 0.000013$ & $0.000087 \pm 0.000301$ & $0.000007 \pm 0.000007$ \\
\hline L & $11.49 \pm 0.25$ & 98.3 & -18.6 & 1108 & $6.55 \mathrm{E}-15$ & 0.43 & $0.093625 \pm 0.000128$ & $0.003350 \pm 0.000026$ & $0.000058 \pm 0.000011$ & $-0.000094 \pm 0.000166$ & $0.000005 \pm 0.000006$ \\
\hline M & $11.59 \pm 0.22$ & 100.3 & -73.9 & 24065 & 7.91E-15 & 0.49 & $0.110820 \pm 0.000112$ & $0.004014 \pm 0.000033$ & $0.000053 \pm 0.000010$ & $-0.000029 \pm 0.000126$ & $-0.000001 \pm 0.000007$ \\
\hline $\mathrm{N}$ & $12.00 \pm 0.23$ & 102.4 & 16.9 & -1020 & $7.94 \mathrm{E}-15$ & 0.56 & $0.108975 \pm 0.000140$ & $0.003892 \pm 0.000026$ & $0.000033 \pm 0.000009$ & $0.000120 \pm 0.000090$ & $-0.000009 \pm 0.000007$ \\
\hline $\mathrm{O}$ & $11.72 \pm 0.21$ & 100.5 & 25.9 & -24205 & $8.49 \mathrm{E}-15$ & 0.62 & $0.118709 \pm 0.000210$ & $0.004258 \pm 0.000028$ & $0.000055 \pm 0.000014$ & $0.000086 \pm 0.000121$ & $-0.000002 \pm 0.000007$ \\
\hline $\mathrm{P}$ & $12.19 \pm 0.32$ & 104.9 & 9.2 & -262 & $5.81 \mathrm{E}-15$ & 0.67 & $0.077774 \pm 0.000117$ & $0.002800 \pm 0.000023$ & $-0.000011 \pm 0.000016$ & $0.000159 \pm 0.000199$ & $-0.000013 \pm 0.000007$ \\
\hline $\mathrm{Q}$ & $12.20 \pm 0.37$ & 105.3 & 6.5 & -888 & $4.78 \mathrm{E}-15$ & 0.71 & $0.063414 \pm 0.000120$ & $0.002289 \pm 0.000025$ & $0.000017 \pm 0.000016$ & $0.000184 \pm 0.000228$ & $-0.000011 \pm 0.000006$ \\
\hline $\mathrm{R}$ & $12.85 \pm 0.39$ & 109.8 & 436.5 & -178 & $4.68 \mathrm{E}-15$ & 0.74 & $0.059895 \pm 0.000103$ & $0.002139 \pm 0.000020$ & $-0.000027 \pm 0.000016$ & $0.000003 \pm 0.000127$ & $-0.000020 \pm 0.000006$ \\
\hline $\mathrm{s}$ & $12.34 \pm 0.33$ & 103.6 & 8.8 & -399 & 5.65E-15 & 0.78 & $0.076670 \pm 0.000130$ & $0.002693 \pm 0.000028$ & $0.000005 \pm 0.000016$ & $0.000160 \pm 0.000184$ & $-0.000009 \pm 0.000007$ \\
\hline
\end{tabular}


$11.64 \pm 0.31$

$11.41 \pm 0.28$

102.6

$5.2-538$

$1.30 \pm 0.28$

1. $28 \mathrm{E}-14$

4. $65 \mathrm{E}-15$

5. $97 \mathrm{E}-15$

$\begin{array}{lll}0.83 & 0.175404 \pm 0.000177 & 0.002695 \pm 0.000024\end{array}$

$\begin{array}{lll}0.86 & 0.067110 \pm 0.000122 & 0.002381 \pm 0.000024\end{array}$

$0.000011 \pm 0.000017$

$5.34 \mathrm{E}-15$

$\begin{array}{lll}0.91 & 0.075680 \pm 0.000113 & 0.002585 \pm 0.00002\end{array}$

$\begin{array}{llll}0.96 & 0.100904 \pm 0.000148 & 0.003075 \pm 0.000026\end{array}$ $0.000036 \pm 0.000015$ Experiment $\# 1870112,0.0017$ $\mathrm{J}=0.00023263423819052 \pm 2.857 \mathrm{E}-07$ $9 / 20 / 18$ $0.000047 \pm 0.000016$ $0.000071 \pm 0.000015$

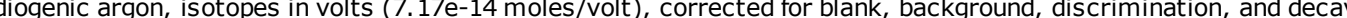
Calculated bulk K/Ca $=13.375 \pm 3.876$, Calculated $\mathrm{K} 2 \mathrm{O}=4.32 \%$ wt. Calculated $\mathrm{CaO}=0.40 \%$ wt. Calculated $\mathrm{Cl}=-5.1 \mathrm{ppm}$

Total Gas Age $=12.4 \pm 8.2 \mathrm{e}-2 \mathrm{Ma}$

Weighted Mean Age $=11.8 \pm 8.5 \mathrm{e}-2 \mathrm{Ma}( \pm 1$ sigma, including $\pm \mathrm{J}), 95.7 \% 39 \mathrm{Ar}$ released

Weighted Mean Age $=11.8 \pm 6.9 \mathrm{e}-2 \mathrm{Ma}$ (a priori, including $\pm \mathrm{J}), 95.7 \%$ 39Ar released

Weighted Mean Age $=11.8 \pm 0.2 \mathrm{Ma}(95 \%$ confidence, including $\pm \mathrm{J})$

MSWD $=1.51$ (Good fit, MSWD < 1.67)

23 of 24 grains

Isochron Age $=11.6 \pm 0.1 \mathrm{Ma}( \pm 1$ sigma, including $\pm \mathrm{J})$

Isochron Age $=11.6 \pm 0.0 \mathrm{Ma}$ (a priori, including $\pm \mathrm{J}$ )

Isochron Age $=11.6 \pm 0.3 \mathrm{Ma}(95 \%$ confidence, including $\pm \mathrm{J})$

$M S W D=10.17$ (Poor fit, MSWD > 1.69)

40Ar/36Ar intercept $=294.0 \pm 7.0( \pm 1$ sigma $)$

$40 \mathrm{Ar} / 36 \mathrm{Ar}$ intercept $=294.0 \pm 2.2$ (a priori)

$40 \mathrm{Ar} / 36 \mathrm{Ar}$ intercept $=294.0 \pm 14.5$ (95\% confidence)

Steps 23 of 24 grains

PSF 17077B Plagioclase (unit MIf)

\begin{tabular}{|c|c|c|c|c|c|c|c|c|c|c|c|}
\hline $\operatorname{emp}\left({ }^{\circ} \mathrm{C}\right.$ & Age(Ma) & $\% 40 \mathrm{Ar} *$ & $\mathrm{~K} / \mathrm{Ca}$ & $\mathrm{K} / \mathrm{Cl}$ & moles 40Ar* & $\sum 39 \mathrm{Ar}$ & $40 \mathrm{Ar}$ & $39 \mathrm{Ar}$ & $38 \mathrm{Ar}$ & $37 \mathrm{Ar}$ & $36 \mathrm{Ar}$ \\
\hline 450 & $12.72 \pm 1.81$ & 56.52 & 0.12 & -329 & $1.20 \mathrm{E}-15$ & 0 & $0.029756 \pm 0.000160$ & $0.002041 \pm 0.000024$ & $0.000009 \pm 0.000015$ & $0.009185 \pm 0.000507$ & $0.000046 \pm 0.000008$ \\
\hline 550 & $19.59 \pm 0.28$ & 76.85 & 0.13 & -880 & $1.66 \mathrm{E}-14$ & 0.02 & $0.304382 \pm 0.000340$ & $0.018389 \pm 0.000061$ & $0.000198 \pm 0.000010$ & $0.073036 \pm 0.001012$ & $0.000256 \pm 0.000011$ \\
\hline 650 & $19.14 \pm 0.09$ & 90.07 & 0.12 & -4970 & 4. $97 \mathrm{E}-14$ & 0.06 & $0.775243 \pm 0.000423$ & $0.056194 \pm 0.000085$ & $0.000744 \pm 0.000031$ & $0.239889 \pm 0.003507$ & $0.000325 \pm 0.000011$ \\
\hline 750 & $18.92 \pm 0.06$ & 93.44 & 0.12 & -9764 & $8.91 \mathrm{E}-14$ & 0.14 & $1.340223 \pm 0.000646$ & $0.101975 \pm 0.000158$ & $0.001361 \pm 0.000046$ & $0.461044 \pm 0.004707$ & $0.000424 \pm 0.000011$ \\
\hline 825 & $18.92 \pm 0.06$ & 94.97 & 0.11 & -10390 & $9.22 \mathrm{E}-14$ & 0.22 & $1.364555 \pm 0.000544$ & $0.105530 \pm 0.000169$ & $0.001398 \pm 0.000046$ & $0.505578 \pm 0.006959$ & $0.000372 \pm 0.000010$ \\
\hline 900 & $18.94 \pm 0.05$ & 95.01 & 0.11 & -87893 & $1.02 \mathrm{E}-13$ & 0.31 & $1.513046 \pm 0.000672$ & $0.116943 \pm 0.000179$ & $0.001592 \pm 0.000038$ & $0.568192 \pm 0.005429$ & $0.000412 \pm 0.000011$ \\
\hline 975 & $18.91 \pm 0.05$ & 96.58 & 0.11 & -4359 & $1.06 \mathrm{E}-13$ & 0.4 & $1.535263 \pm 0.000644$ & $0.120845 \pm 0.000189$ & $0.001516 \pm 0.000046$ & $0.590804 \pm 0.003585$ & $0.000342 \pm 0.000009$ \\
\hline 1050 & $18.89 \pm 0.05$ & 95.46 & 0.11 & -12961 & $1.09 \mathrm{E}-13$ & 0.49 & $1.611571 \pm 0.000611$ & $0.125504 \pm 0.000154$ & $0.001668 \pm 0.000032$ & $0.606362 \pm 0.003562$ & $0.000415 \pm 0.000010$ \\
\hline 1100 & $18.89 \pm 0.06$ & 95.04 & 0.11 & 7366 & $9.12 \mathrm{E}-14$ & 0.57 & $1.339420 \pm 0.000615$ & $0.103867 \pm 0.000159$ & $0.001479 \pm 0.000029$ & $0.508898 \pm 0.002938$ & $0.000366 \pm 0.000010$ \\
\hline 1150 & $19.01 \pm 0.06$ & 92.29 & 0.11 & -7609 & $8.43 \mathrm{E}-14$ & 0.65 & $1.283207 \pm 0.000479$ & $0.096000 \pm 0.000136$ & $0.001281 \pm 0.000044$ & $0.477674 \pm 0.003193$ & $0.000466 \pm 0.000011$ \\
\hline 1225 & $19.09 \pm 0.06$ & 84.14 & 0.11 & 26580 & 1.09E-13 & 0.74 & $1.825524 \pm 0.000623$ & $0.123968 \pm 0.000165$ & $0.001845 \pm 0.000030$ & $0.610659 \pm 0.006514$ & $0.001141 \pm 0.000015$ \\
\hline 1300 & $19.15 \pm 0.04$ & 89.31 & 0.11 & -19918 & $1.92 \mathrm{E}-13$ & 0.91 & $3.025262 \pm 0.000912$ & $0.217390 \pm 0.000259$ & $0.003037 \pm 0.000045$ & $1.047256 \pm 0.003050$ & $0.001378 \pm 0.000014$ \\
\hline 1325 & $19.34 \pm 0.06$ & 86.9 & 0.11 & -12813 & 1.07E-13 & 1 & $1.734071 \pm 0.000658$ & $0.120037 \pm 0.000147$ & $0.001693 \pm 0.000039$ & $0.583170 \pm 0.005767$ & $0.000925 \pm 0.000014$ \\
\hline
\end{tabular}

Packet IRR367-CG, Experiment \#18Z0051, $0.0879 \mathrm{~g}$ Plagioclase, all errors \pm 1 sigma

J $=0.0 \quad 6 / 19 / 18$

40Ar* is radiogenic argon, isotopes in volts (7.12e-14 moles/volt), corrected for blank, background, discrimination, and decay

Calculated bulk K/Ca $=0.109 \pm 3.453 \mathrm{e}-2$, Calculated $\mathrm{K} 2 \mathrm{O}=0.48 \%$ wt., Calculated $\mathrm{CaO}=5.34 \%$ wt., Calculated $\mathrm{Cl}=-4.6 \mathrm{e}-2 \mathrm{ppm}$

Total Gas Age $=19.02 \pm 2.21 \mathrm{e}-2 \mathrm{Ma}$

Weighted Mean Plateau Age $=18.91 \pm 2.57 e-2 \mathrm{Ma}( \pm 1$ sigma, including $\pm \mathrm{J}), 51.61 \%$ 39Ar released
$0.000270 \pm 0.000118$

$-0.000034 \pm 0.000206$

$0.000101 \pm 0.000128$

$0.000081 \pm 0.000127$

$0.000080 \pm 0.00015$

$0.000008 \pm 0.000006$

$0.000013 \pm 0.000006$

$0.000057 \pm 0.000007$

$0.000007 \pm 0.000006$ 
Weighted Mean Plateau Age $=18.91 \pm 2.57 \mathrm{e}-2 \mathrm{Ma}$ (A priori, including $\pm \mathrm{J}), 51.61 \%$ 39Ar released

Weighted Mean Plateau Age $=18.91 \pm 2.33 \mathrm{e}-2 \mathrm{Ma}(95 \%$ confidence, including $\pm \mathrm{J})$

MSWD $=0.17$ (Good fit, MSWD < 2.56)

Steps 6 of $13\left(750,825,900,975,1050,1100^{\circ} \mathrm{C}\right)$

Isochron Age $=18.9 \pm 0.1 \mathrm{Ma}( \pm 1$ sigma, including $\pm \mathrm{J})$

Isochron Age $=18.9 \pm 0.1 \mathrm{Ma}$ (A Priori Errors, including $\pm \mathrm{J}$ )

Isochron Age $=18.9 \pm 0.3 \mathrm{Ma}(95 \%$ confidence, including $\pm \mathrm{J})$

MSWD $=0.19$ (Good fit, MSWD < 2.77)

40Ar/36Ar intercept $=306.5 \pm 35.0( \pm 1$ sigma $)$

$40 \mathrm{Ar} / 36 \mathrm{Ar}$ intercept $=306.5 \pm 35.0$ (A Priori)

40Ar/36Ar intercept $=306.5 \pm 77.9$ ( $95 \%$ confidence $)$

Steps 6 of $13\left(750,825,900,975,1050,1100^{\circ} \mathrm{C}\right)$

PSF 17052 Basalt (unit OMI)

\begin{tabular}{|c|c|c|c|c|c|c|c|c|c|c|c|}
\hline $\mathrm{mp}\left({ }^{\circ} \mathrm{C}\right.$ & Age(Ma) & $\% 40 \mathrm{Ar}^{*}$ & $\mathrm{~K} / \mathrm{Ca}$ & $\mathrm{K} / \mathrm{Cl}$ & moles 40Ar* & $\sum 39 \mathrm{Ar}$ & $40 \mathrm{Ar}$ & $39 \mathrm{Ar}$ & $38 \mathrm{Ar}$ & $37 \mathrm{Ar}$ & $36 \mathrm{Ar}$ \\
\hline 450 & $18.42 \pm 0.73$ & 32.69 & 0.29 & -421 & $5.60 \mathrm{E}-15$ & 0 & $0.240910 \pm 0.000208$ & $0.006532 \pm 0.000038$ & $0.000121 \pm 0.000024$ & $0.011902 \pm 0.000426$ & $0.000546 \pm 0.000010$ \\
\hline 500 & $18.32 \pm 0.28$ & 50.28 & 0.32 & 7493 & 1. $99 \mathrm{E}-14$ & 0.02 & $0.555012 \pm 0.000331$ & $0.023269 \pm 0.000061$ & $0.000493 \pm 0.000034$ & $0.038584 \pm 0.000617$ & $0.000935 \pm 0.000014$ \\
\hline 550 & $17.62 \pm 0.16$ & 61.67 & 0.36 & -3142 & 3. $27 \mathrm{E}-14$ & 0.05 & $0.745827 \pm 0.000317$ & $0.039873 \pm 0.000086$ & $0.000650 \pm 0.000014$ & $0.058812 \pm 0.001348$ & $0.000974 \pm 0.000014$ \\
\hline 600 & $16.59 \pm 0.13$ & 67.5 & 0.42 & -2478 & 3. $76 \mathrm{E}-14$ & 0.09 & $0.782139 \pm 0.000361$ & $0.048627 \pm 0.000109$ & $0.000715 \pm 0.000028$ & $0.060765 \pm 0.000874$ & $0.000868 \pm 0.000013$ \\
\hline 650 & $16.22 \pm 0.14$ & 44.33 & 0.46 & -2499 & 5. $29 \mathrm{E}-14$ & 0.14 & $1.677114 \pm 0.000627$ & $0.070022 \pm 0.000125$ & $0.001386 \pm 0.000027$ & $0.080494 \pm 0.001448$ & $0.003150 \pm 0.000021$ \\
\hline 700 & $16.05 \pm 0.05$ & 80.11 & 0.43 & -3059 & 1. $13 \mathrm{E}-13$ & 0.25 & $1.983796 \pm 0.000604$ & $0.151259 \pm 0.000158$ & $0.002027 \pm 0.000065$ & $0.185134 \pm 0.001584$ & $0.001374 \pm 0.000015$ \\
\hline 750 & $15.70 \pm 0.03$ & 93.96 & 0.39 & -5890 & $1.53 \mathrm{E}-13$ & 0.4 & $2.294720 \pm 0.000584$ & $0.209852 \pm 0.000235$ & $0.002698 \pm 0.000032$ & $0.284297 \pm 0.001938$ & $0.000544 \pm 0.000011$ \\
\hline 800 & $15.75 \pm 0.03$ & 95.5 & 0.37 & -6283 & $1.62 \mathrm{E}-13$ & 0.56 & $2.379862 \pm 0.000879$ & $0.220557 \pm 0.000203$ & $0.002822 \pm 0.000042$ & $0.312524 \pm 0.001180$ & $0.000447 \pm 0.000011$ \\
\hline 850 & $15.71 \pm 0.04$ & 89.92 & 0.36 & -32239 & 1. $30 \mathrm{E}-13$ & 0.69 & $2.024705 \pm 0.000670$ & $0.177116 \pm 0.000189$ & $0.002438 \pm 0.000031$ & $0.256909 \pm 0.002053$ & $0.000756 \pm 0.000013$ \\
\hline 925 & $15.64 \pm 0.04$ & 85.52 & 0.39 & -21349 & $1.50 \mathrm{E}-13$ & 0.84 & $2.468500 \pm 0.000682$ & $0.206306 \pm 0.000211$ & $0.002900 \pm 0.000030$ & $0.275551 \pm 0.001707$ & $0.001275 \pm 0.000015$ \\
\hline 1000 & $15.46 \pm 0.04$ & 88.5 & 0.27 & 4315 & $1.21 \mathrm{E}-13$ & 0.96 & $1.923286 \pm 0.000666$ & $0.168360 \pm 0.000174$ & $0.002526 \pm 0.000029$ & $0.332136 \pm 0.001632$ & $0.000834 \pm 0.000013$ \\
\hline 1100 & $15.27 \pm 0.10$ & 83.53 & 0.06 & 1207 & 4. $00 \mathrm{E}-14$ & 1 & $0.673173 \pm 0.000419$ & $0.056559 \pm 0.000127$ & $0.001023 \pm 0.000030$ & $0.464623 \pm 0.002931$ & $0.000502 \pm 0.000011$ \\
\hline 1200 & $10.74 \pm 2.56$ & 48.69 & 0.12 & 79 & 7.47E-16 & 1 & $0.021573 \pm 0.000119$ & $0.001500 \pm 0.000032$ & $0.000108 \pm 0.000019$ & $0.006295 \pm 0.000217$ & $0.000039 \pm 0.000008$ \\
\hline
\end{tabular}

Packet IRR367-CI, Experiment \#18Z0046, $0.0386 \mathrm{~g}$ Basalt, all errors \pm 1 sigma

$J=0.0 \quad 6 / 4 / 18$

40Ar* is radiogenic argon, isotopes in volts (7.12e-14 moles/volt), corrected for blank, background, discrimination, and decay

Calculated bulk K/Ca $=0.305 \pm 0.109$, Calculated $\mathrm{K} 2 \mathrm{O}=1.15 \%$ wt., Calculated $\mathrm{CaO}=4.61 \%$ wt., Calculated $\mathrm{Cl}=-9.7 \mathrm{e}-2 \mathrm{ppm}$

Total Gas Age $=15.86 \pm 2.06 \mathrm{e}-2 \mathrm{Ma}$

Weighted Mean Plateau Age $=15.71 \pm 2.74 \mathrm{e}-2 \mathrm{Ma}( \pm 1$ sigma, including $\pm J), 58.99 \%$ 39Ar released

Weighted Mean Plateau Age $=15.71 \pm 2.00 \mathrm{e}-2 \mathrm{Ma}$ (A priori, including $\pm \mathrm{J}), 58.99 \% 39 \mathrm{Ar}$ released

Weighted Mean Plateau Age $=15.71 \pm 6.02 \mathrm{e}-2 \mathrm{Ma}(95 \%$ confidence, including $\pm \mathrm{J})$

MSWD $=1.88($ Good fit, MSWD < 3.12)

Steps 4 of $13\left(750,800,850,925^{\circ} \mathrm{C}\right)$

Isochron Age $=15.8 \pm 0.04 \mathrm{Ma}( \pm 1$ sigma, including $\pm \mathrm{J})$

Isochron Age $=15.8 \pm 0.04 \mathrm{Ma}(\mathrm{A}$ Priori Errors, including $\pm \mathrm{J})$

Isochron Age $=15.8 \pm 0.1 \mathrm{Ma}(95 \%$ confidence, including $\pm \mathrm{J})$

MSWD $=0.61$ (Good fit, MSWD < 3.69)

$40 \mathrm{Ar} / 36 \mathrm{Ar}$ intercept $=285.5 \pm 6.6( \pm 1$ sigma $)$

$40 \mathrm{Ar} / 36 \mathrm{Ar}$ intercept $=285.5 \pm 6.6$ (A Priori)

$40 \mathrm{Ar} / 36 \mathrm{Ar}$ intercept $=285.5 \pm 14.7(95 \%$ confidence $)$ 
Steps 4 of $13\left(750,800,850,925^{\circ} \mathrm{C}\right)$ 